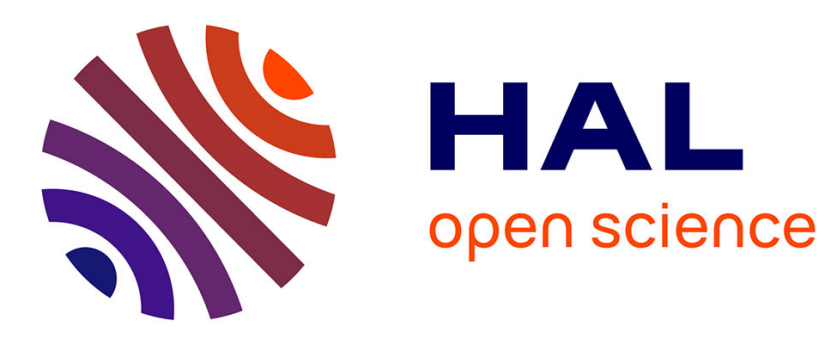

\title{
Sinking a Granular Raft
}

Suzie Protière, Christophe Josserand, Jeffrey M. Aristoff, Howard A. Stone, Manouk Abkarian

\section{To cite this version:}

Suzie Protière, Christophe Josserand, Jeffrey M. Aristoff, Howard A. Stone, Manouk Abkarian. Sinking a Granular Raft. Physical Review Letters, 2017, 118 (10), pp.108001. 10.1103/PhysRevLett.118.108001 . hal-01513620

\section{HAL Id: hal-01513620 https: / hal.sorbonne-universite.fr/hal-01513620}

Submitted on 25 Apr 2017

HAL is a multi-disciplinary open access archive for the deposit and dissemination of scientific research documents, whether they are published or not. The documents may come from teaching and research institutions in France or abroad, or from public or private research centers.
L'archive ouverte pluridisciplinaire HAL, est destinée au dépôt et à la diffusion de documents scientifiques de niveau recherche, publiés ou non, émanant des établissements d'enseignement et de recherche français ou étrangers, des laboratoires publics ou privés. 


\title{
Sinking a Granular Raft
}

\author{
Suzie Protière, ${ }^{1}$ Christophe Josserand, ${ }^{1}$ Jeffrey M. Aristoff, ${ }^{2}$ Howard A. Stone, ${ }^{3}$ and Manouk Abkarian ${ }^{4}$ \\ ${ }^{1}$ CNRS UMR 7190, Sorbonne Universités, UPMC Univ Paris 06, Institut Jean Le Rond d'Alembert, F-75005 Paris, France \\ ${ }^{2}$ Numerica Corporation, 5042 Technology Parkway, Suite 100, Fort Collins, Colorado 80528, USA \\ ${ }^{3}$ Department of Mechanical and Aerospace Engineering, Princeton University, Princeton, New Jersey 08544, USA \\ ${ }^{4}$ CNRS UMR 5048, University Montpellier, Centre de Biochimie Structurale, 34090 Montpellier, France
}

(Received 25 July 2016; revised manuscript received 4 November 2016; published 6 March 2017)

\begin{abstract}
We report experiments that yield new insights on the behavior of granular rafts at an oil-water interface. We show that these particle aggregates can float or sink depending on dimensionless parameters taking into account the particle densities and size and the densities of the two fluids. We characterize the raft shape and stability and propose a model to predict its shape and maximum length to remain afloat. Finally we find that wrinkles and folds appear along the raft due to compression by its own weight, which can trigger destabilization. These features are characteristics of an elastic instability, which we discuss, including the limitations of our model.
\end{abstract}

DOI: 10.1103/PhysRevLett.118.108001

Archimedes discovered the force of buoyancy over two thousand years ago. He stated in his famous principle that the buoyancy of an object is strictly related to the weight of fluid displaced by its immersed part. However, at millimeter scales, effective buoyancy is significantly influenced by capillary forces, which become dominant at the colloidal scale. A general Archimedes principle can thus be formulated where flotation is guaranteed only if the weight of the immersed body or particle remains smaller than the weight of the fluid displaced over the entire deformed meniscus surrounding it $[1,2]$. These local capillary deformations induce monopolar and long-range interactions between different particles straddling an interface [3], leading to the formation of an aggregate referred to as a raft in the literature. An apparent paradoxical and critical situation can then be observed: while each particle is able to float owing to the generalized Archimedean principle, the stability of the entire raft is in fact not guaranteed [4]. Capillary deformations overlap, producing a global deformation of the raft that can lead to its sinking. It is difficult to predict a priori the critical shape of this assembly of particles because of the nonlocality and loss of large scale additivity of capillary interactions. In previous work [5], we focused on how the destabilization of a raft induces the production of particlearmored droplets, but the general conditions for flotation of a granular raft and its dynamics of destabilization have not yet been explored.

Granular rafts are unique composite materials that resist tension due to capillarity and resist compression due to the steric repulsion between grains. While rafts can deform similarly to elastic sheets [6], displaying wrinkling instabilities when compressed [7], they also display granular properties such as local fluidization [8] or jamming $[9,10]$ unique to these interfacial structures. More generally, understanding the mechanical properties of these particle-laden interfaces is key since they can be observed and used in many natural situations $[11,12]$ and have found countless innovative and broad range technological applications, such as in encapsulation [13,14], flotation [15], emulsion [16], and remediation processes [5], in surface functionalization $[17,18]$, or for the production of new materials [13,19-21]. In this Letter, we investigate the behavior of a buoyancydriven assembly of dense athermal grains, and study the global shape, stability, and dynamics of granular rafts.

A container is filled with water on top of which an oil layer of a few millimeters thick is deposited. Heavy grains are then sprinkled carefully from above. We denote $\rho_{w}, \rho_{o}$, and $\rho_{s}$ the mass density of water, oil, and grains, respectively, and introduce the capillary length $\ell_{c}=\sqrt{\gamma /\left(\rho_{w}-\rho_{o}\right) g}$, with $\gamma$ being the oil-water interfacial tension. The particles pierce the air-oil interface and finally straddle the oil-water interface where they remain trapped. We studied the raft shape using ceramic zirconium oxide beads (Glen Mills, Inc.) of various compositions $\left(\rho_{s}=3.8 \mathrm{~g} \mathrm{~cm}^{-3}\right.$ or $\rho_{s}=6 \mathrm{~g} \mathrm{~cm}^{-3}$ ) and diameters $b=250-900 \mu \mathrm{m}$ and established a stability diagram using grains of other materials such as Delrin $\left(\rho_{s}=1.4 \mathrm{gcm}^{-3}, b=3.17 \mathrm{~mm}\right)$, stainless steel $\left(\rho_{s}=8 \mathrm{gcm}^{-3}\right.$, $b=1 \mathrm{~mm})$, and teflon $\left(\rho_{s}=2.5 \mathrm{~g} \mathrm{~cm}^{-3}, b=900 \mu \mathrm{m}\right)$. Since the particles are heavy, they create a strong deformation of the interface. Surface tension causes the deformed surface area to be minimized and thus forces the particles to move towards each other and form aggregates (Fig. 1). Particles are sprinkled in order to create a nearly axisymmetric raft (Fig. 1). As particles are added progressively, the raft becomes denser, deforms, and starts bowing in the middle, displacing a large amount of liquid in this region (Fig. 1).

The evolution of the cross-sectional shape of a granular raft as particles are added uniformly to its edge can be quantified primarily by measuring its maximum depth $h(r=0) / \ell_{c}$ as a function of its maximum radial extension 


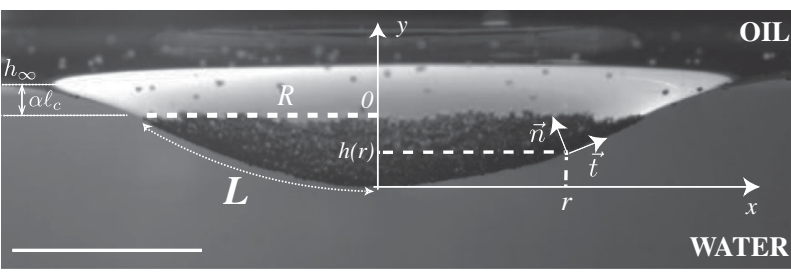

FIG. 1. (a) Side view and notations for a granular raft at the oil-water interface. $\mathcal{D}_{1}=11.5, \mathcal{D}_{2}=1.95$ [defined in Eq. (6)]. Scale bar: $1 \mathrm{~cm}$.

$r=R$ (Fig. 1). Two different behaviors are observed depending on the diameter of the particles. First (i.e., $b=900 \mu \mathrm{m}$ ), the raft deepens nonlinearly and sharply for small variations of its lateral extension. Its shape remains concave until $h(0)$ reaches $\ell_{c}$ where sinking occurs [Fig. 2(a)]. For smaller particles (e.g., $b=500 \mu \mathrm{m}$ ), the raft becomes larger in radius, but the critical depth before sinking still remains close to $\ell_{c}$. However, in the second type of behavior, for even smaller particles (e.g., $b=350 \mu \mathrm{m}$ ), we still observe an increase of $h(0)$ but only up to an inflection point at $R / \ell_{c} \sim 1$, represented by $A$ on Fig. 2(a). From $A$, the slope decreases and almost vanishes up to a second point labeled $B$ on Fig. 2(a). From there, $h$ increases again linearly with $R$ up to a point $C$ where sinking occurs at a depth larger than $\ell_{c}$. In Fig. 2(b), the three different profiles associated to $A, B$, and $C$ are reported. Until $A$, the raft remains concave as in the first behavior but the bottom of the raft starts to flatten [Fig. 2(b), profile $A]$. When more particles are added, the flattened region extends and we observe the development of undulations on its surface [Fig. 2(b), profile $B$ ]. From the point $B$, one of these protrusions deepens and, at the point $C$ just before sinking, the raft is almost flat everywhere except for a large fold scarring its surface. In fact, the linear variation of $h(0)$ between points $B$ and $C$ [Fig. 2(a)] describes the growth of the protrusion.

In order to describe the evolution of the depth and the shape of the raft, we propose a minimal model considering the granular raft as a floating, axisymmetric, continuous, heavy sheet of thickness $b$ at equilibrium. A volume fraction $\Phi$ of the sheet is composed of grains of size $b$, a material of density $\rho_{s}$, and the complementary volume of a material with a density $\left(\rho_{w}+\rho_{o}\right) / 2$ (particles are assumed to be equally immersed in both phases and always in contact between each other so that the volume fraction is larger than a minimum value $\Phi_{\min }>0$ ). This sheet is subjected to three different forces: the weight of the grains, the hydrostatic pressure due both to the oil and water displacements of the interface, and a tension $\vec{T}(r)$ that accounts both for the liquid interface that is still present, the contact force between the grains, and the lateral pressure force due to the two liquids in the sheet of thickness $b$. This latter contribution is due to the finite thickness $b$ of the sheet and disappears in the limit $b \rightarrow 0$ in contrast with the surface tension. Since the grains can easily slide along each other, we assume that the shear modulus due to the grain contacts in the sheet can be neglected compared to the bulk modulus as a first approximation [22]. This leads to an isotropic tension $\vec{T}=T(r) \vec{t}$ (where $\vec{t}$ is the unit tangent vector to the raft surface; see Fig. 1), and the normal and tangential force balances respectively give

$$
\begin{aligned}
& T^{\prime}(r)-\left(\Phi \rho_{s}+(1-\Phi) \frac{\rho_{w}+\rho_{o}}{2}\right) b g h^{\prime}(r)=0, \text { and } \\
& T(r)\left(\frac{h^{\prime \prime}}{1+h^{\prime 2}}+\frac{h^{\prime}}{r}\right)+\left(\rho_{w}-\rho_{o}\right) g\left(h_{\infty}-h\right) \sqrt{1+h^{\prime 2}} \\
& \quad+g b \Phi\left(\frac{\rho_{w}+\rho_{o}}{2}-\rho_{s}\right)=0,
\end{aligned}
$$

where $h(r)$ denotes the height of the interface $\left(h_{\infty}\right.$ indicates the height of the oil-water interface at infinity; see Fig. 1). This set of equations is valid in the domain where the floating sheet is present, i.e., for $r \leq R$ where $R$ is the raft
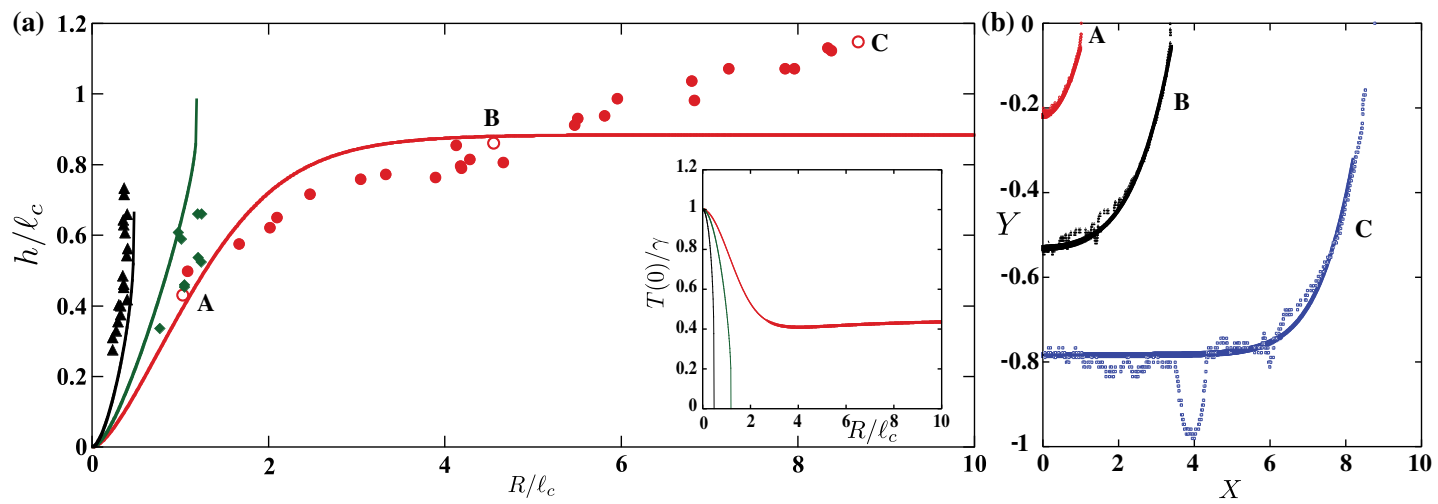

FIG. 2. (a) Depth of the raft as a function of $R$. Inset: Tension $T(0)$ at the center of the raft as its perimeter is increased (see model). (triangle), $b=900 \mu \mathrm{m}\left(\mathcal{D}_{1}=3.75, \mathcal{D}_{2}=0.9\right)$; (diamond), $b=500 \mu \mathrm{m}\left(\mathcal{D}_{1}=2.08, \mathcal{D}_{2}=0.5\right) ;($ circle $), b=350 \mu \mathrm{m}\left(\mathcal{D}_{1}=1.46\right.$, $\mathcal{D}_{2}=0.35$ ). (b) Experimental and theoretical [Eq. (5)] profiles of raft $\mathcal{D}_{1}=1.46$ and $\mathcal{D}_{2}=0.35$, as its perimeter increases, using the rescaled variables $X=x / \ell_{c}$ and $Y=y / \ell_{c}$. The open symbols in (a) correspond the raft profiles A, B and C displayed in (b). 
radius (Fig. 1). It is worth noting that the limit $\Phi=0$ (not possible for grains in contact however) or similarly $\rho_{s}=$ $\left(\rho_{o}+\rho_{w}\right) / 2$ leads to a residual term in Eq. (1) that accounts for the variation of the pressure within the sheet. For $r>R$ (the pure interface beyond the raft), the interface is determined by the usual equation of equilibrium for a fluid-fluid interface [by taking $b=0$ in Eqs. (1) and (2)],

$\gamma\left(\frac{h^{\prime \prime}}{1+h^{\prime 2}}+\frac{h^{\prime}}{r}\right)+\left(\rho_{w}-\rho_{o}\right) g\left(h_{\infty}-h\right) \sqrt{1+h^{\prime 2}}=0$.

Boundary conditions are determined by the geometry, $h^{\prime}(0)=0$ and $\lim _{r \rightarrow \infty} h(r)=h_{\infty}$, and also by the value of the tension at the raft edge $T(R)=\gamma$. Remarkably, in this model, the contact force plays the same role as the surface tension: it is increasing with $r$ because of the compression caused by the weight of the grains, so that the effective tension $T(r)$ (corresponding to the surface tension $\gamma$ minus the compression due to the weight) is decreasing from $r=R$ towards the center $r=0$. We propose that stability of the structure is then obtained as long as the effective surface tension $T(r)$ is positive, which yields a simple criterion for the existence and stability of the floating sheet, i.e., $T(0)>0$.

Next, the length scales are rescaled using $\ell_{c}$ and tension with $\gamma$, giving the dimensionless system,

$$
\begin{gathered}
T^{\prime}-\left(\Phi \mathcal{D}_{1}+(1-\Phi) \mathcal{D}_{2}\right) h^{\prime}=0 \\
T\left(\frac{h^{\prime \prime}}{1+h^{\prime 2}}+\frac{h^{\prime}}{r}\right)+\left(h_{\infty}-h\right) \sqrt{1+h^{\prime 2}}+\Phi\left(\mathcal{D}_{2}-\mathcal{D}_{1}\right)=0 .
\end{gathered}
$$

Here we have introduced two dimensionless numbers that control the system,

$$
\mathcal{D}_{1}=\frac{\rho_{s}}{\left(\rho_{w}-\rho_{o}\right)} \frac{b}{\ell_{c}} \quad \text { and } \quad \mathcal{D}_{2}=\frac{\left(\rho_{w}+\rho_{o}\right) / 2}{\left(\rho_{w}-\rho_{o}\right)} \frac{b}{\ell_{c}} \text {. }
$$

Both $\mathcal{D}_{1}$ and $\mathcal{D}_{2}$ compare the weight acting at the scale of the sheet thickness and the counterbalancing buoyancy at the scale of the raft. Here, $\mathcal{D}_{1}$ considers the part of the sheet composed of a material of density $\rho_{s}$ (as introduced for floating sheets [2]) and $\mathcal{D}_{2}$ the part with a density $\rho_{w}+\rho_{o} / 2$. Since the grains are more dense than the two liquids, we always have $\mathcal{D}_{1}>\mathcal{D}_{2}$.

The shape of the raft $h(r)$ is calculated using a relaxation scheme that converges to a static solution for given values of $\mathcal{D}_{1}$ and $\mathcal{D}_{2}$. Specifically, a virtual dynamics is performed by introducing a term $\alpha \partial_{\tau} h$ on the right-hand side of Eq. (5), where $\tau$ is a virtual time. The convergence towards the equilibrium solution is very rapid and a fixed criterion is used on the variation of the solution to determine the raft profile. When a negative tension $T$ is reached (first at $r=0$ ) the dynamics becomes erratic indicating that the raft is unstable.

In Fig. 2(a), we plot the solutions $h(0)$ for different $\mathcal{D}_{1}$ and $\mathcal{D}_{2}$. The trend of the first behavior (i.e., the evolution of rafts of large particles) is well captured by our model with only one fitting parameter $\Phi=0.75$ for all particle sizes. For the smaller diameter particles, we reproduce the initial evolution of the curve up to the typical point $B$. However, from this point the model predicts a constant depth with an infinitely long and flat raft that we do not observe experimentally [compare profiles in Fig. 2(b)]. Such constant depth solution can be easily inferred from the model and corresponds to a balance between the raft weight and the hydrostatic pressure, leading to $h_{\infty}=\Phi\left(\mathcal{D}_{1}-\mathcal{D}_{2}\right)$.

Also, our model gives the variation of the effective tension along the raft and during its growth. The inset of Fig. 2(a) represents the evolution of $T(0)$ as the raft grows for the three different particle sizes, exhibiting again two different regimes: for the largest particles, the tension decreases toward 0 for a finite value of $R$, while for the smallest ones, $T(0)$ reaches a plateau. Subsequently, the value for which $T(0)$ vanishes defines the critical size for a floating raft to exist, beyond which it should become unstable and sink. While the numerics recover correctly this critical raft size observed experimentally for the grain diameters $b=900 \mu \mathrm{m}$ and $b=500 \mu \mathrm{m}$, the model fails to describe the instability of the smaller particles $(b=350 \mu \mathrm{m})$, which seems to have another origin.

Indeed, beyond stability, sinking is controlled by the shape of the cross section of the raft at the critical radius. We thus observe two types of destabilization. For the largest particle sizes, the raft sinks in a single encapsulating event producing one armored droplet with a diameter on the scale of the raft [inset (i), Fig. 3(a), Supplemental Material [23] movie 1]. However, for the smallest particle sizes, the growth of a deep enough protrusion destabilizes the raft into an interfacial granular jet that displays a RayleighPlateau-like instability and breaks into numerous armored droplets. The typical size of these droplets is much smaller than the raft extension and is comparable to the jet diameter [inset (ii), Fig. 3(a), Supplemental Material [23] movie 2].

Next, we organize our results in a diagram that illustrates how large a raft can grow before sinking [Fig. 3(a)]. We plot half of a raft's maximum normalized curvilinear and cross-sectional length, $\tilde{L}=L / \ell_{c}$, as a function of $\mathcal{D}_{1}$ and $\mathcal{D}_{2}$. For $\mathcal{D}_{1}>3$, rafts are small compared to $\ell_{c}$. The larger the $\mathcal{D}_{1}$, the smaller the raft can extend before sinking occurs as a single large armored droplet [inset Fig. 3(a)]. In this region, similarly to the one-dimensional approach studied in [4], all the data fall onto a single power law in $\mathcal{D}_{1}^{-1}$ nearly independent of $\mathcal{D}_{2}$. An asymptotic analysis of Eqs. (4) and (5) captures such a limiting behavior for sinking by considering that the raft exhibits a parabolic profile, leading to $\tilde{L} \propto 1 /\left(\Phi \mathcal{D}_{1}\right)$. For $\mathcal{D}_{1}<3$ however, $\tilde{L}$ can be as large as 20. In this region, $\tilde{L}$ varies rapidly and is sensitive to $\mathcal{D}_{2}$. 




FIG. 3. (a) Floating to sinking transition of a granular raft: $\tilde{L}=L / \ell_{c}$, for varying $\mathcal{D}_{1}$ and $\mathcal{D}_{2}$. (circle) $\mathcal{D}_{2}<0.5$, (triangle) $0.5<\mathcal{D}_{2}<1$, (diamond) $1<\mathcal{D}_{2}<2.5$, and (square) $\mathcal{D}_{2}>2.5$. Dashed lines present the analytical float-sink transitions for $\mathcal{D}_{2}=0.5$ (red), $\mathcal{D}_{2}=1$ (blue), and $\mathcal{D}_{2}=2.5$ (green). Inset: Time lapse presenting the two possible types of destabilization. (i) $t=0 \mathrm{~s}$, $t \approx 100 \mathrm{~ms}, t \approx 200 \mathrm{~ms}, \mathcal{D}_{1}=6.93, \mathcal{D}_{2}=2.84$. (ii) $t=0 \mathrm{~s}, t \approx 200 \mathrm{~ms}, t \approx 400 \mathrm{~ms}, \mathcal{D}_{1}=11.5$, $\mathcal{D}_{2}=1.95$. (b) Raft, $\mathcal{D}_{1}=2.74$, $\mathcal{D}_{2}=0.71$, (plus) right before sinking and during destabilization, and (c) raft $\mathcal{D}_{1}=3.84, \mathcal{D}_{2}=1$ (asterisk) right before sinking and during its destabilization. (d) Transition for raft $\mathcal{D}_{1}=17.3$ and $\mathcal{D}_{2}=2.84$ aggregated (filled star) axisymmetrically or (e) (open star) in line.

The larger the $\mathcal{D}_{2}$, the larger the raft can grow for a given $\mathcal{D}_{1}$.

In fact, our model predicts that infinite rafts can exist for each $\mathcal{D}_{1}$ for low enough $\mathcal{D}_{2}$. More precisely, the infinite raft domain is delimited by a boundary $\mathcal{D}_{1}^{*}\left(\mathcal{D}_{2}\right)$, in the $\left(\mathcal{D}_{1}, \mathcal{D}_{2}\right)$ parameter space, which can be computed by considering that the height of the interface meniscus between the raft and the infinite interface (at $h_{\infty}$ ) is $\alpha \ell_{c}$,

$$
\mathcal{D}_{1}^{*}\left(\mathcal{D}_{2}\right)=D_{2}+\frac{\alpha-\mathcal{D}_{2}+\sqrt{\left(\alpha-\mathcal{D}_{2}\right)^{2}+4\left(\alpha \mathcal{D}_{2}+1\right)}}{2 \Phi} .
$$

An infinite raft can exist [in the sense that $T(0)>0$ ] for $\mathcal{D}_{1}>\mathcal{D}_{1}^{*}\left(\mathcal{D}_{2}\right)$. This boundary is in good agreement with the experimental observations, taking as a fitting parameter $\alpha=0.75$ and using $\Phi=0.75$ again (Supplemental Material [23]). Remarkably, $\mathcal{D}_{1}^{*}(0)=\left(\alpha+\sqrt{\alpha^{2}+4}\right) /(2 \Phi)$ indicating that for $\mathcal{D}_{1}<\mathcal{D}_{1}^{*}(0)$ the model predicts no sinking, whatever the value of $\mathcal{D}_{2}$. This behavior has also been predicted in a one-dimensional approach by Vella et al. [7]. Lastly, as explained above, this curve only characterizes the possibility for the existence of an infinite raft in the sense of exhibiting a positive tension but it says nothing about other possible dynamical instabilities such as buckling that could prevent the existence of an infinite raft. Indeed, we observe that even for $\mathcal{D}_{1} \lesssim 3$, rafts may develop wrinkles during their growth [Fig. 3(b)]. A large fold progressively grows and the wrinkles flatten. For a depth of the order of $\ell_{c}$, the fold transforms into a narrow granular jet that drains the entire raft [inset (ii), Fig. 3(a)
Supplemental Material [23] movie 2]. This buckling instability thus prevents here the existence of an infinite raft. By simplifying the behavior of the granular raft to that of a continuous sheet bearing only tension due to grain contacts and surface tension, we are still able to describe its behavior and stability. This is true especially for large $\mathcal{D}_{1}$ (and $\mathcal{D}_{2}$ ) where we would expect such a continuous approach to fail since in this region rafts are made only of a few particles. Our model suggests that granular rafts, not too large relative to $\ell_{c}$, remain in tension, even close to the sinking transition. Both $\mathcal{D}_{1}$ and $\mathcal{D}_{2}$ are necessary to describe the raft's stability. These parameters define how the forces acting at the scale of single particles are related to the behavior of the entire raft. Though we used many different materials and batches of grains with varying degrees of polydispersity and contact angles, the data in Fig. 3(a) are still gathered around a universal law captured by our model.

The model offers thus a comprehensive approach to raft sinking although it also has some limitations. First, it is limited to axisymmetric situations, as shown in Figs. 3(d) and 3(e), where we produce two rafts of different shapes (axisymmetric or in line), keeping everything else constant, and find two different $\tilde{L}$. Secondly, the model fails to describe the metastability seen for $\mathcal{D}_{1}<3$ [Fig. 3(b)]. For intermediate $\mathcal{D}_{1} \sim 3$, the raft develops a $d$-cone structure [Fig. 3(c)] just before sinking. Such features are found in elastic sheets put under compression [24,25], where bending elasticity would be the essential ingredient for their description. This suggests that for $\mathcal{D}_{1} \lesssim 3$, regions of compression 
appear during the raft growth due to the added weight of the particles at its boundary. A first step to explain the presence of these compressive zones could be to consider the existence of a shear resistance in the granular sheet with an apparent shear modulus. The resulting model bears inherently anisotropic tensions that depend on the radial position in the raft, delimiting regions of compression close to the center and tension dominated ones at the border. The raft resists such compressive forces by bending out of plane, since an isotropic biaxial bending moment can then be defined [22], though an expression and mechanism for such granular bending resistance is still debated [7,26,27].

The constitutive law defining the behavior of this composite interfacial material is far from trivial. The effective material properties should be expected to vary along the sheet. While the effective tension arises from the interfacial tension, compression forces are borne by grainto-grain interactions. The possibility for grains to rearrange during tension phases gives this material a unique plastic nature [8] not yet accounted for but that could explain the jetting transition we observe experimentally. In order to fully characterize the mechanics of such interfacial composite materials, and, in particular, its possible buckling instability, the next step is to derive a full granular and elastic model including the raft's ability to bend and wrinkle under compression. But whatever the origin of this elasticity, our experiments show at last that not only an object's tension and weight governs its stability against sinking but another general key ingredient is its elasticity.

[1] J. B. Keller, Phys. Fluids 10, 3009 (1998).

[2] D. Vella, Annu. Rev. Fluid Mech. 47, 115 (2015).

[3] A. Dominguez, M. Oettel, and S. Dietrich, J. Chem. Phys. 128, 114904 (2008).

[4] D. Vella, P. D. Metcalfe, and R. J. Whittaker, J. Fluid Mech. 549, 215 (2006).

[5] M. Abkarian, S. Protière, J. M. Aristoff, and H. A. Stone, Nat. Commun. 4, 1895 (2013).

[6] P. M. Reis, J. Hure, S. Jung, J. W. M. Bush, and C. Clanet, Soft Matter 6, 5705 (2010).
[7] D. Vella, P. Aussillous, and L. Mahadevan, Europhys. Lett. 68, 212 (2004).

[8] A. Subramaniam, M. Abkarian, L. Mahadevan, and H. A. Stone, Langmuir 22, 10204 (2006).

[9] M. Abkarian, A. B. Subramaniam, S. H. Kim, R. J. Larsen, S. M. Yang, and H. A. Stone, Phys. Rev. Lett. 99, 188301 (2007).

[10] O. Pitois, M. Buisson, and X. Chateau, Eur. Phys. J. E 38, 48 (2015).

[11] J. C. Loudet, A. M. Alsayed, J. Zhang, and A. G. Yodh, Phys. Rev. Lett. 94, 018301 (2005).

[12] N. Mlot, C. Tovey, and D. Hu, Proc. Natl. Acad. Sci. U.S.A. 108, 7669 (2011).

[13] Y. Xia, B. Gates, Y. Yin, and Y. Lu, Adv. Mater. 12, 693 (2000).

[14] B. P. Binks and R. Murakami, Nat. Mater. 5, 865 (2006).

[15] M. Caballero, R. Cela, and J. A. Perez-Bustamante, Talanta 37, 275 (1990).

[16] J. W. Tavacoli, G. Katgert, E. G. Kim, M. E. Cates, and P. S. Clegg, Phys. Rev. Lett. 108, 268306 (2012).

[17] R. A. W. Dryfe, Phys. Chem. Chem. Phys. 8, 1869 (2006).

[18] Z. Zhang, C. Geng, Z. Hao, T. Wei, and Q. Yan, Adv. Colloid Interface Sci. 228, 105 (2016).

[19] M.-H. Wu and G. M. Whitesides, Appl. Phys. Lett. 78, 2273 (2001).

[20] D. T. Wasan and A. D. Nikolov, Nature (London) 423, 156 (2003).

[21] K. Stratford, R. Adhikari, I. Pagonabarraga, J. Desplat, and M. Cates, Science 309, 2198 (2005).

[22] D. C. Pamplona and C. R. Calladine, J. Biomech. Eng. 115, 149 (1993).

[23] See Supplemental Material http://link.aps.org/supplemental/ 10.1103/PhysRevLett.118.108001 for the movies showing the sinking of the rafts presented in the inset of Fig. 3a and details concerning the model.

[24] S. Chaïeb, F. Melo, and J.-C. Géminard, Phys. Rev. Lett. 80, 2354 (1998).

[25] E. Cerda and L. Mahadevan, Proc. R. Soc. A 461, 671 (2005).

[26] C. Planchette, E. Lorenceau, and A.-L. Biance, Soft Matter 8, 2444 (2012).

[27] T. D. Kassuga and J. P. Rothstein, J. Colloid Interface Sci. 448, 287 (2015). 